\title{
Instrumentação para medidas de mobilidade eletrônica e concentração de portadores em amostras semicondutoras, pelo método de van der Pauw
}

\author{
Instrumentation for electronic mobility and carrier concentration measurements in semiconductor \\ samples, using the van der Pauw method \\ Instrumentación para medidas de movilidad electrónica y concentración de portadores en muestras \\ de semiconductores, mediante el método de van der Pauw
}

Recebido: 19/04/2021 | Revisado: 16/05/2021 | Aceito: 17/05/2021 | Publicado: 04/06/2021

Luiz Henrique Ribeiro

ORCID: https://orcid.org/0000-0002-0723-0170 Universidade Federal de Itajubá, Brasil E-mail: luizhenriquerib@yahoo.com.br

Adhimar Flávio Oliveira

ORCID: https://orcid.org/0000-0003-2586-7359 Universidade Federal de Itajubá, Brasil E-mail: adhimarflavio@unifei.edu.br

Rero Marques Rubinger

ORCID: https://orcid.org/0000-0003-1718-9658 Universidade Federal de Itajubá, Brasil E-mail: rero@unifei.edu.br

\begin{abstract}
Resumo
Neste trabalho apresentamos os resultados da construção de um equipamento, de baixo custo, para medidas medida Hall, utilizando a técnica de van der Pauw. Para isso, foi desenvolvido um sistema composto por uma placa eletrônica controlada por uma placa DAQ que juntamente com um software em Labview realiza a automação de todas as medidas necessárias. O sistema é capas de realizar todas as operações necessárias entre os contatos para realizar a técnica de van der Pauw e obter parâmetros como mobilidade eletrônica, concentração de portadores e resistividades elétrica em função da temperatura. Tais medidas são essenciais para estudar o comportamento eletrônico de amostras, visando sua aplicação em dispositivos eletrônicos. Para verificar o comportamento do sistema, foi utilizado uma amostra de Indium tin oxide (ITO). Nesses testes verificou-se que o os tempos de sincronização utilizados para as medidas apresentam resultados com baixo ruído e que a montagem permite a realização de medidas didáticas para disciplinas de pósgraduação e também de pesquisas desenvolvidas pelos alunos de pós-graduação.
\end{abstract}

Palavras-chave: Método de van der Pauw; Mobilidade eletrônica; Concentração de portadores; Semicondutores; Automação.

\begin{abstract}
In this work we present the results of the construction of low-cost equipment for Hall measurements, using the van der Pauw technique. For this, a system was developed composed of an electronic board controlled by a DAQ board that together with software in Labview performs the automation of all necessary measures. The system is capable of carrying out all the necessary switch operations between the contacts to perform the van der Pauw technique and obtain parameters such as electronic mobility, carriers concentration, and electrical resistivities as a function of temperature. Such measures are essential to study the electronic behavior of samples, aiming at their application in electronic devices. To check the behavior of the system, a sample of Indium tin oxide (ITO) was used. In these tests, it was found that the synchronization times used for the measurements show results with low noise and that the assembly allows the carrying out of didactic measures for undergraduate courses and also for research carried out by graduate students.
\end{abstract}

Keywords: Van der Pauw method; Electronic mobility; Concentration of carriers; Semiconductors; Automation.

\section{Resumen}

En este trabajo presentamos los resultados de la construcción de un equipo de bajo costo para medidas Hall, utilizando la técnica de van der Pauw. Para ello, se desarrolló un sistema compuesto por una placa electrónica controlada por una placa DAQ que junto con el software en Labview realiza la automatización de todas las medidas necesarias. El sistema es capaz de realizar todas las operaciones necesarias entre los contactos para realizar la técnica de van der Pauw y obtener parámetros como movilidad electrónica, concentración de portadores y resistividades eléctricas en función de la temperatura. Estas medidas son fundamentales para estudiar el comportamiento electrónico de las muestras, con el 
objetivo de su aplicación en dispositivos electrónicos. Para comprobar el comportamiento del sistema se utilizó una muestra de óxido de indio y estaño (ITO). En estas pruebas se encontró que los tiempos de sincronización utilizados para las mediciones arrojan resultados con bajo nivel de ruido y que el montaje permite la realización de medidas didácticas para cursos de posgrado y también para investigaciones realizadas por estudiantes de posgrado.

Palabras clave: Método de Van der Pauw; Movilidad electrónica; Concentración de portadores; Semiconductores; Automatización.

\section{Introdução}

Tecnologias para a produção de materiais que estão envolvidos na geração de dispositivos eletrônicos, tem atingido enorme avanço nos últimos anos (Sousa et. al. 2021; Martins et. al. 2020). Materiais semicondutores compostos principalmente dos grupos II-VI e dos grupos III-V da tabela periódica, têm sido usados na fabricação de dispositivos eletrônicos de alta eficiência, tais como os de potência e alta frequência (Adachi, 2005; Sun et al. 2014; Zhang \& David 2021). Os dispositivos atuais precisam possuir cada vez mais menor dimensionalidade e podem ser encontrados em manufaturas de dispositivos como circuitos integrados, diodos emissores de luz infravermelhos, diodos laser, LEDs detectores luminosos e células solares (Wróbel et. al, 2020; Liu et al. 2015).

Dentre os materiais semicondutores desenvolvidos com compostos dos grupos II-IV e III-V o Arseneto de Gálio (GaAs) e o Arseneto de Índio (InAs) tem se destacado devido ao seu bandgap direto a alta mobilidade eletrônica em comparação com outros semicondutores, como o silício $(\mathrm{Si})$, maior resistência à radiação ionizante, e outras propriedades elétricas e ópticas superiores à do Si (Oliveira, 2015; Toledo, 2020). Estruturalmente eles apresentam a forma cúbica cristalina do tipo zincblende, sendo muito importantes na indústria microeletrônica, interessantes na fabricação de dispositivos optoelétricos de alta eficiência (Jiang, 2018) particularmente para a fabricação de dispositivos que interajam com a luz e a eletricidade.

A mobilidade dos portadores de carga em um cristal depende das interações desses portadores com os átomos da rede cristalina, com os átomos de impurezas, com defeitos da rede, com outros portadores, etc. Todas essas interações são conhecidas por mecanismos de espalhamento (Kraus et al. 2021).

O estudo da mobilidade eletrônica é de grande importância na investigação e compreensão dos mecanismos de espalhamento que afetam a dinâmica do movimento dos portadores em materiais semicondutores (Sze et al., 2021, Guo et. Al. 2021; Feng et. al. 2020). As investigações das características do comportamento eletrônico visam ampliar a gama de aplicações destes materiais.

Neste trabalho apresentamos os resultados de uma pesquisa experimental (Pereira et.al. 2018) da construção de um equipamento, de baixo custo, para medidas medida Hall, utilizando a técnica de van der Pauw. Para isso, foi desenvolvida uma placa de reles que é controlada via placa DAQ através de um software em Labview. Todo o sistema foi concebido no laboratório de Caracterização de Materiais (LCM) do Instituto de Física e Química (IFQ), pelos alunos de pós-gradução. Além de apresentar a montagem instrumental e o software de controle, são apresentados resultados de uma medida realizada numa amostra teste, através do qual foi possível verificar os tempos de sincronização das medidas e o controle de temperatura. Atualmente este equipamento está sendo utilizado nas aulas do programa de pós-graduação em Materiais para Engenharia e por alunos de pósgraduação em seus trabalhos.

\section{Metodologia}

\subsection{Efeito Hall}

Considere um semicondutor no formato de uma barra ilustrada na Figura 1. Após a aplicação do campo magnético na direção $z$, os elétrons experimentam uma força $-e \cdot \boldsymbol{v} \boldsymbol{x} \boldsymbol{B}$, que terá uma componente $\mathrm{y}-e \cdot(\boldsymbol{v} \boldsymbol{x} \boldsymbol{B})_{\mathrm{y}}, \log \mathrm{o}:-e\left(v_{z} B_{x}-v_{x} B_{z}\right)=$ $e \cdot v_{x} B_{z}$. A carga elétrica se acumulará na face $+\mathrm{y}$ da amostra até que uma força oposta $-e \cdot E_{y}$ entre em equilíbrio com a força 
$e \cdot v_{x} B_{x}$. A densidade de corrente total é, naturalmente, $j_{x}=-n e v_{x}$, de modo que $e \cdot E_{y}=e v_{x} \cdot B=-e B \cdot j_{x} / n e$. O coeficiente Hall $R_{H}$ é definido pela equação 1 .

$R_{H}=\frac{E_{Y}}{j_{X} B}=-\frac{1}{n \cdot e}$

Figura 1: Configuração para medidas de resistividade e Efeito Hall onde $L_{s}$ é o comprimento, w a largura e d a espessura da barra. Pelo comprimento da barra é percorrida uma corrente elétrica $\mathrm{I}$, em que $\mathrm{V}_{\mathrm{H}}$ é medida uma diferença de potencial denominada de tensão Hall, e $\mathrm{V}_{\mathrm{c}}$ é a tensão de condução.

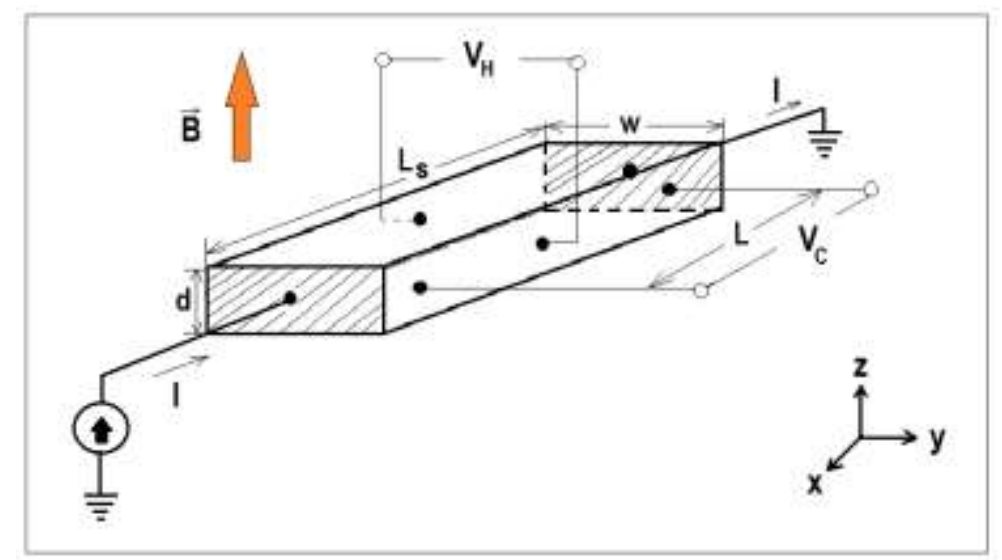

Fonte: Autores (2021).

Sendo a equação 1 válida para a maioria dos materiais semicondutores e, em particular o GaAs. Em uma análise mais precisa o coeficiente Hall é dado pela equação 2.

$R_{H}=-\frac{r}{n \cdot e}$

em que $r$ é o fator Hall. Para baixos valores de $E$, a velocidade $v_{X}$ varia linearmente com $E_{X}$, isto é, $v_{X}=\mu E_{X}$. O coeficiente de proporcionalidade entre $v$ e $E$, chamado de mobilidade $\mu$. A mobilidade pode então ser medida da seguinte forma:

$\mu=\frac{v_{X}}{E_{X}}=-\frac{j_{X}}{n e E_{X}}=\frac{j_{X}}{E_{X}} \frac{E_{Y}}{j_{X} B}=\frac{1}{B} \frac{E_{Y}}{E_{X}}=\frac{1}{B}\left(\frac{V_{C}}{V_{H}} \frac{w}{l}\right)^{-1}=\frac{1}{B} \frac{V_{H}}{V_{C}} \frac{l}{w}$

em que $V_{C}=E_{X} l$ é a tensão de condução, ou seja, a tensão entre os contatos paralelos para o fluxo de corrente, e $V_{H}=E_{Y} w$ é a tensão entre os contatos perpendiculares para o fluxo de corrente, a tensão Hall. As equações 1 e 3 constituem as equações para o experimento Hall. A condutividade é definida pela equação 4.

$\sigma=n e \mu$,

onde $\sigma$ é a condutividade. Quando todos os elétrons têm a mesma magnitude da velocidade no eixo x, a mobilidade Hall $\mu_{H}=\mu$, e da equação 1 temos que $R_{H}=-1 / n_{H} e$, onde $n_{H}$ é a concentração de portadores Hall.

\subsection{Método de van der Pauw}

A caracterização elétrica de um material semicondutor é muito importante, pois traz informações sobre resistividade, condutividade, energia de ativação, a mobilidade eletrônica e a concentração de portadores de carga (Wolfe et al. 1989). Durante 
este procedimento é importante saber as dimensões da amostra do material investigado, porém, nem sempre as amostras possuem formas regulares, o que pode dificultar os cálculos de suas propriedades. Uma forma de contornar esse problema é através do método van der Pauw (Oliveira et. al.2021). O método de van der Pauw é o mais utilizado para as medições de resistência elétrica em materiais semicondutores, por ser um método que independe do formato da amostra, sendo necessário apenas a sua espessura. Além disso, tal método contorna vários erros. Van der Pauw formulou essa técnica em 1958 para evitar os erros como desalinhamento dos contatos, efeito Seebeck, e resolver problemas de potencial (Mosbah et. al. 2021; Look, 1992)

A caracterização com 4 pontos utilizando o método de van der Pauw, na qual são realizadas medidas de resistividade pela inclusão da permutação entre os quatro contatos, dois são usados para a aplicação de uma corrente elétrica, e os outros dois na leitura de uma tensão, conforme pode ser visto na Figura 2.

Figura 2: Forma arbitrária de uma amostra indicando os contatos para a medida de (a) resistividade e (b) coeficiente Hall, através da técnica de van der Pauw. Na Figura 2 é a corrente elétrica e Vc a tensão de condução.
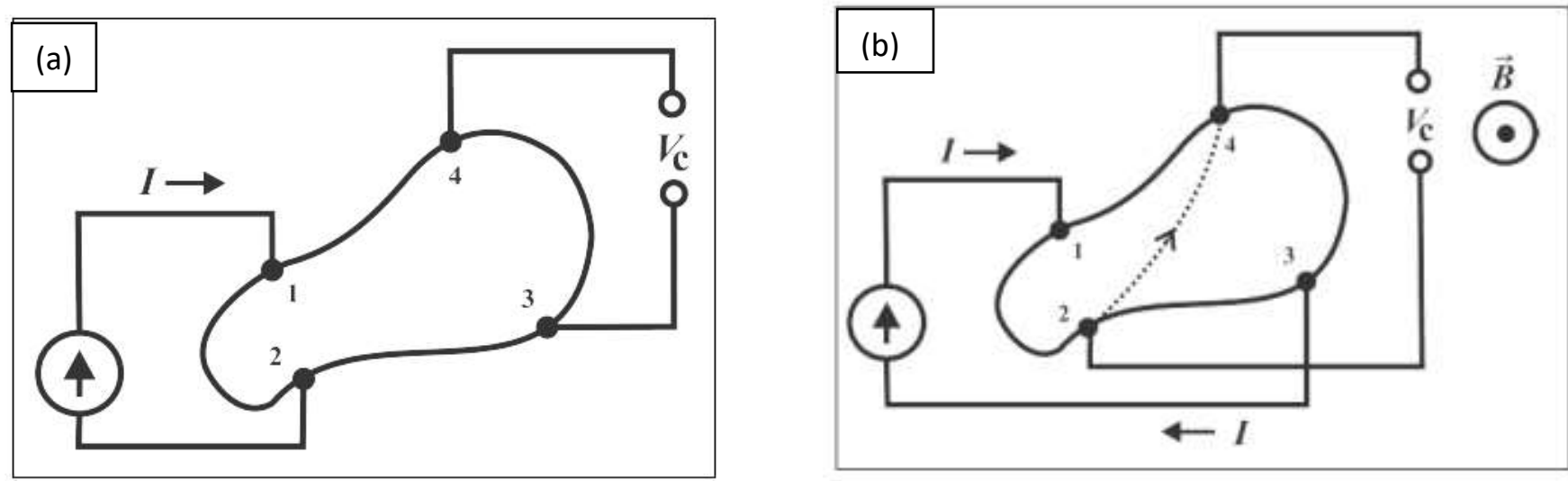

Fonte: Autores (2021).

Seja $R_{i j, k l}=V_{k l} / I_{i j}$, onde a corrente entra no contato i e sai pelo contato j, e $V_{k l}=V_{k}-V_{l}$. A resistividade, na ausência de campo magnético é dada pela Equação 5.

$\rho=\frac{\pi d}{\ln 2} \cdot\left[\frac{R_{21,34}+R_{32,41}}{2}\right] \cdot f$

em que $f$ é determinado da equação transcendental:

$\frac{Q-1}{Q+1}=\frac{f}{\ln 2} \cdot \operatorname{arccosh}\left\{\frac{1}{2} \cdot \exp \left[\frac{\ln 2}{f}\right]\right\}$

em que $Q=R_{21,34} / R_{32,41}$ se essa relação for maior que a unidade, caso contrário $Q=R_{32,41} / R_{21,34}$. Uma curva de $f$ vs $Q$, com precisão de 2\%, é apresentada na Figura 3. 
Figura 3: Função de taxa de resistividade usada para corrigir os resultados de van der Pauw para a forma de amostra assimétrica.

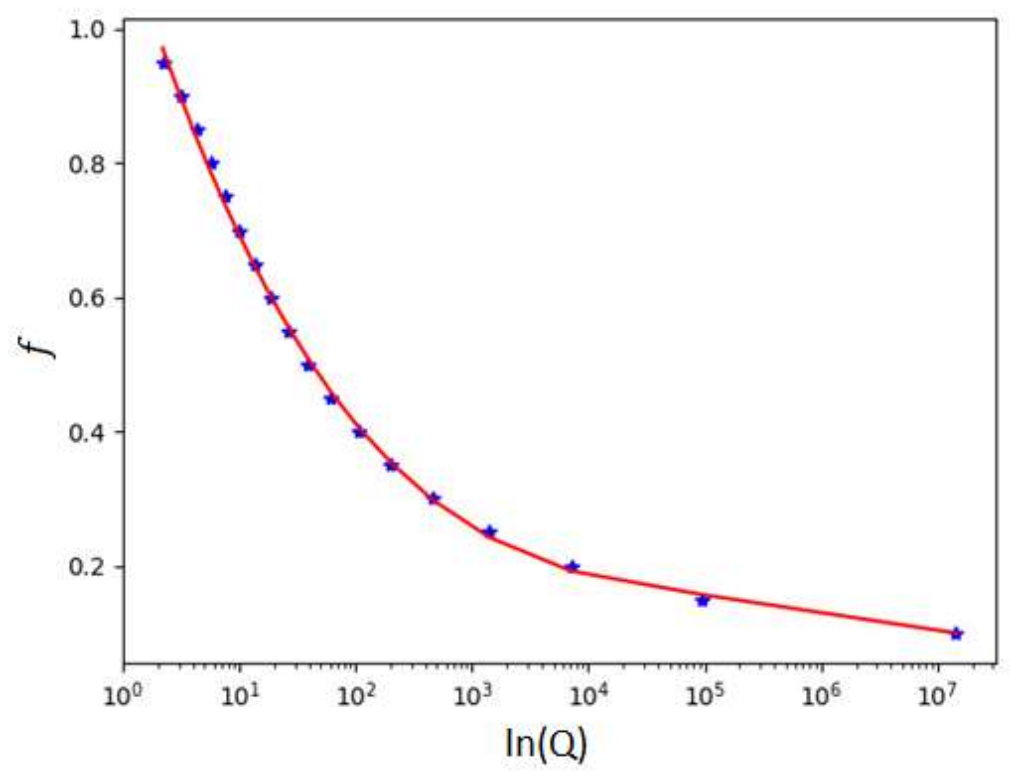

Fonte: Autores (2021).

A resistividade $\rho$ pela técnica de van de Pauw é dada por:

$\rho=\frac{\pi d}{\ln 2} \cdot \frac{1}{8}\left(R_{21,34}-R_{12,34}+R_{32,41}-R_{23,41}\right) \cdot f_{A}+$

$\frac{\pi d}{\ln 2} \cdot \frac{1}{8}\left(R_{43,12}-R_{34,12}+R_{14,23}-R_{41,23}\right) \cdot f_{B}$

onde $f_{A}$ e $f_{B}$ são determinados de $Q_{A}$ e $Q_{B}$ respectivamente através das fórmulas abaixo.

$Q_{A}=\frac{R_{21,34}-R_{12,34}}{R_{32,41}-R_{23,41}}$

$Q_{B}=\frac{R_{43,12}-R_{34,12}}{R_{14,23}-R_{41,23}}$

A mobilidade Hall é determinada usando a configuração da Figura 2(b), a qual a corrente e a tensão são cruzadas. Na presença de um campo magnético, para semicondutores isotrópicos, leva à seguinte equação:

$\boldsymbol{E}=\rho \boldsymbol{j}+\rho \mu_{H}(\boldsymbol{j} \times \boldsymbol{B})$

O cálculo do coeficiente Hall foi realizado pela equação 11. Para minimizar a magnetoresistência e outros efeitos é necessária uma média do campo magnético corrente e o campo com a polaridade invertida.

$R_{H}=\frac{d}{B} \cdot \frac{1}{8}\left(R_{31,42}(+B)-R_{13,42}(+B)+R_{42,13}(+B)-R_{24,13}(+B)\right)+$

$\frac{d}{B} \cdot \frac{1}{8}\left(R_{13,42}(-B)-R_{31,42}(-B)+R_{24,13}(-B)-R_{42,13}(-B)\right)$ 


\subsection{Desenvolvimento e testes da instrumentação}

Para o desenvolvimento de uma pesquisa de pós-graduação no programa de Materiais para Engenhara e para disciplinas do mesmo programa foi desenvolvido um sistema automatizado de medidas Hall pela técnica de van de Pauw no laboratório de Laboratório de Caracterização de Materiais (LCM), do Instituto de Física e Química (IFQ). Para a realização das medições foram utilizados uma Source Meter Keithley 2400, um multímetro, também da marca Keithley 2100, uma placa DAQ, uma fonte de alta corrente da marca GMW, um eletroímã, um sistema com criostato e outros equipamentos. Todo o sistema foi automatizado para a coleta automática dos dados. Para isso, foi utilizado o programa que permitiu a realização de todas as operações de variação da temperatura, inversão de campo magnético, ativação do campo elétrico e medida de corrente, apresentação de gráficos em tempo real e arquivamento dos dados para análise posterior. O esquema de montagem da instrumentação é apresentado na Figura 4.

Figura 4: Esquema de montagem das fontes, juntamente com o multímetro e o controle de temperatura do criostato para realização de medidas na amostra.

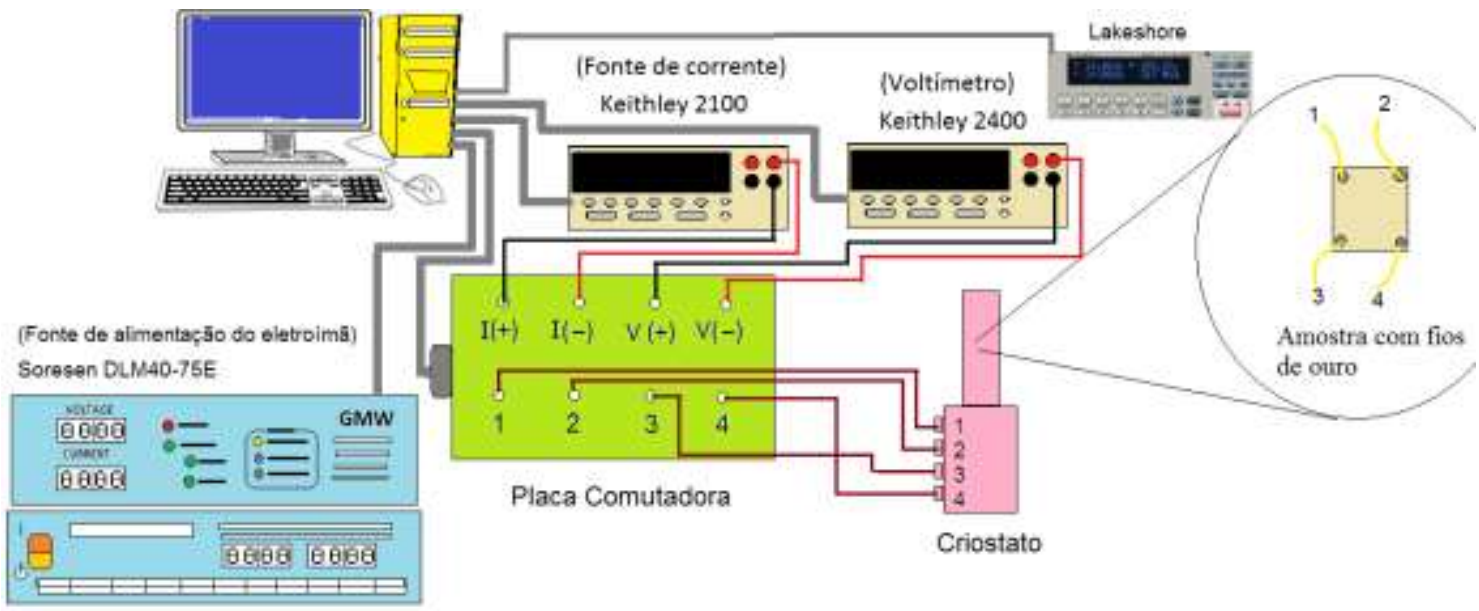

Fonte: Autores (2021).

O programa desenvolvido em Labview inicializa simultaneamente os equipamentos Keithley 2400 e 2100, Lakeshore e a fonte de eletroímã GMW. Para isso, são enviados os valores de temperatura inicial, temperatura final e o passo de varredura das temperaturas, para a criação de um vetor de temperaturas que é enviado para controlar a Lakeshore. São enviados também dados de corrente para a fonte GMW para controle do campo magnético aplicado sobre a amostra. Na fonte Keithley 2400 é configurado o limite máximo de corrente.

Após o envio do valor de temperatura para realizar a medida, o programa analisa constantemente se os sensores de temperatura na amostra estão nos valores desejados. Senão o processo continua até que o valor de temperatura, dentro de uma faixa de erro seja obtido. Após obter esse valor o programa segue para a etapa de medições. Esse processo se repete para todos os valores de temperatura enviados na inicialização do programa. Um fluxograma de funcionamento do programa é apresentado na Figura 5. 
Figura 5: Fluxograma de funcionamento do programa, desenvolvido em Labview, para medidas de resistividade, mobilidade e concentração de portadores. No fluxograma é possível verificar as etapas com e sem a aplicação de campo magnético.

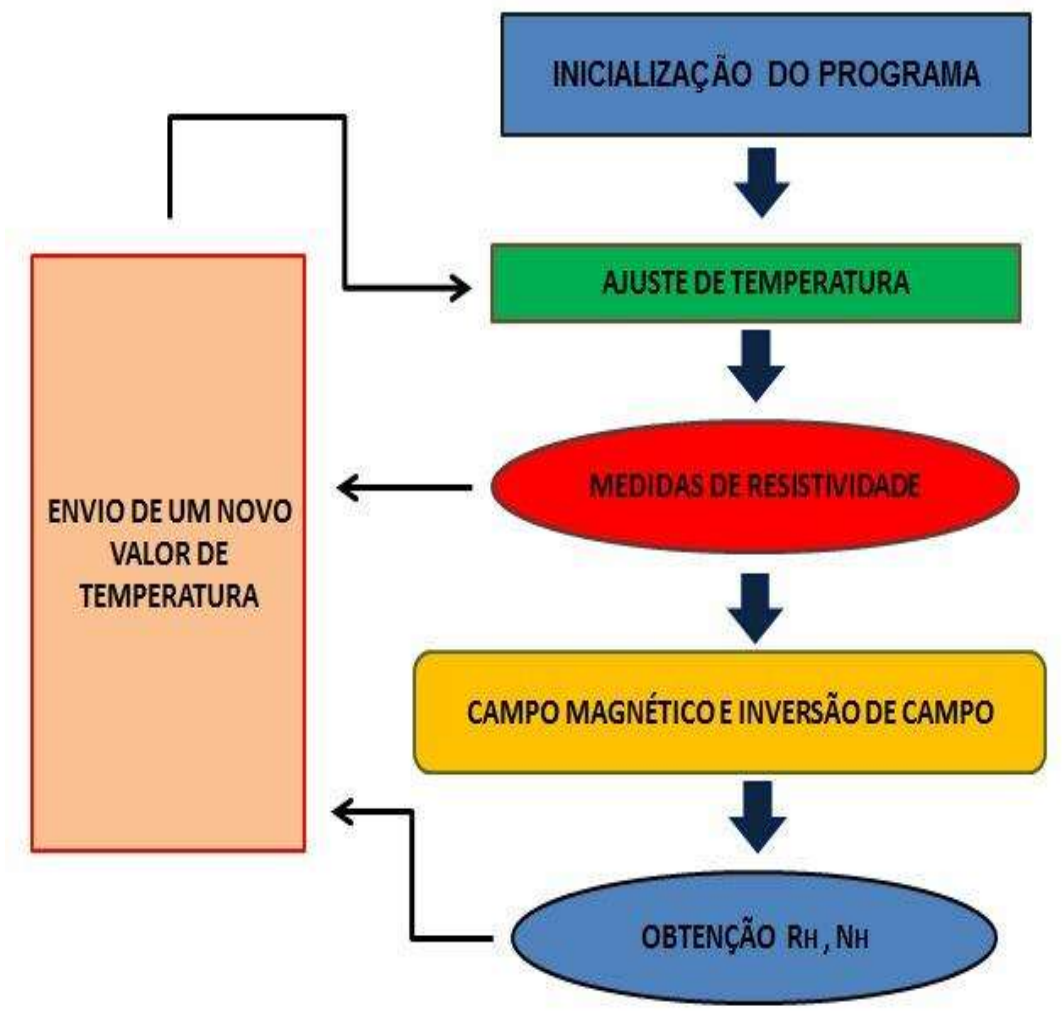

Fonte: Autores (2021).

O programa envia o comando para a placa DAQ, que controla os relés da placa Hall (Figura 6) e realiza todas as operações de medida de resistividade e $R_{H}$ pela técnica de van der Pawn para aquele valor de temperatura. Esse valor é apresentado em um gráfico na tela do usuário em função da temperatura. Além disso, os dados são salvos em arquivos de dados, para análise posterior. Nesse trabalho apresentaremos os resultados para a medida de uma amostra Indium tin oxide (ITO) (Dietrich et. al. 2021). 
Figura 6: Foto da placa de comutação entre os contatos da amostra. Esta placa é controlada por uma placa DAQ, que por sua vez é controlada pelo software desenvolvido em LabView. Além do controle dessa placa a placa DAQ, juntamente com o software, controla também as mudanças nos valores de campo magnético aplicados na amostra.

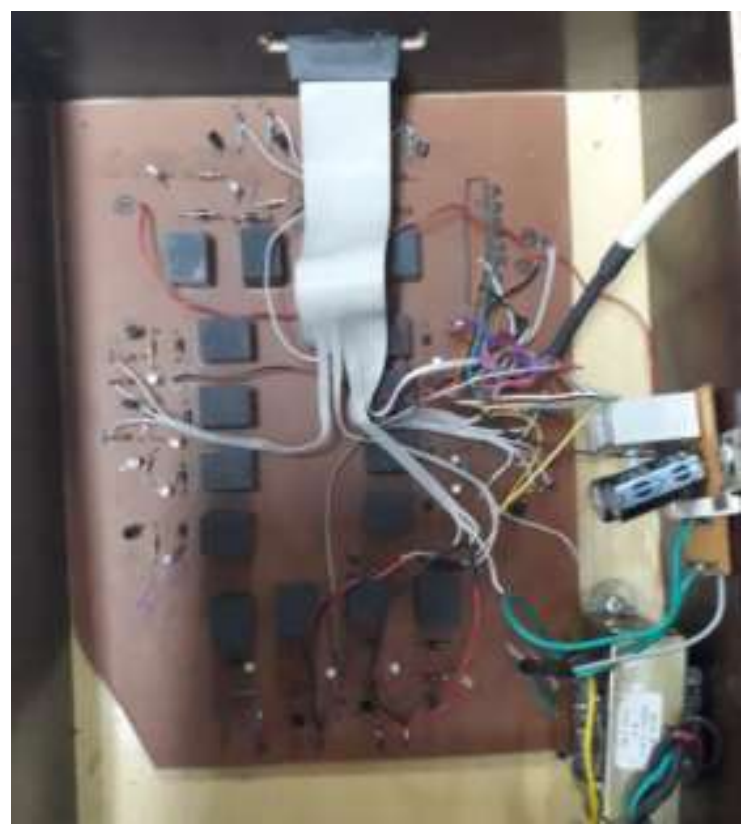

Fonte: Autores (2021).

\section{Resultados e Discussão}

Na Figura 8 é apresentado o gráfico da medida de resistividade em função da temperatura. A amostra varia sua resistividade de $1,08 \times 10^{-6} \Omega \mathrm{m}$, em $10 \mathrm{~K}$, para $1,24 \times 10^{-6} \Omega \mathrm{m}$ na temperatura ambiente. Esse aumento da resistividade com a temperatura é típico de amostras metálicas, ou seja, que possui uma sobreposição entre as bandas de valência e condução [1]. Podemos verificar na medida que o equipamento se comportou bem. O intervalo de comutação escolhido para os relés, juntamente com a aplicação de corrente da Keithley 2400 e a medida de tensão pela Keithley 2100, está correto, ou seja, o sistema teve uma boa sincronização. Além disso, foi possível verificar que o software desenvolvido em Labview controlou corretamente a variação de temperatura. Como essa foi uma medida teste os intervalos de temperatura foram adotados com $5 \mathrm{~K}$, mas o software permite estipular intervalos de até $1 \mathrm{~K}$. Baixos valores no passo de variação de temperatura são importantes para amostras semicondutores, nas quais serão extraídas informações em baixos valores de temperatura. Uma variação pequena de temperatura vai permitir o sistema dentro do criostato ter maior estabilidade, deixando as medidas ainda mais confiáveis. 
Figura 7: Resistividade da amostra de ITO utilizada para verificar o comportamento do instrumento entre a temperatura ambiente e a temperatura de $10 \mathrm{~K}$. Podemos verificar pela resistividade que essa amostra apresentou um comportamento metálico.

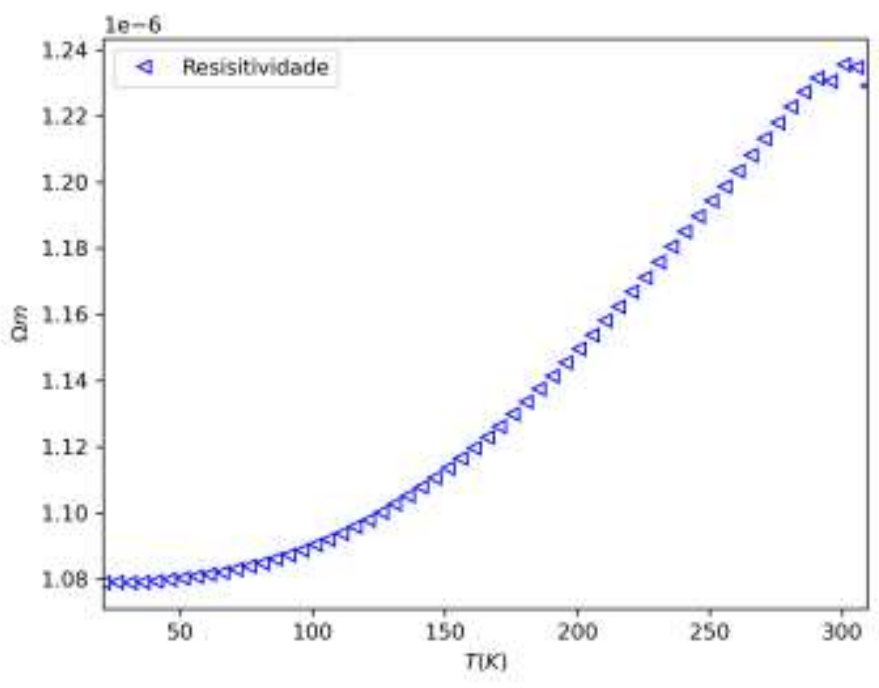

Fonte: Autores (2021).

Na Figura 8 é apresentado o resultado da medida de concentração de portadores em função da temperatura. Como pode ser verificado essa concentração é aproximadamente constante e da ordem de $3 \times 10^{27} \mathrm{~m}^{-3}$. Tal resultado indica que a amostra possui apenas um canal de condução e não existem defeitos capturando os portadores.

Figura 8: Concentração de portadores em função da temperatura para amostra de ITO. No gráfico podemos verificar que a amostra possui uma quantidade aproximadamente constante de portadores, da ordem de $3 \times 10^{27} \mathrm{~m}^{-3}$. Indicando que a amostra possui apenas um canal de condução.

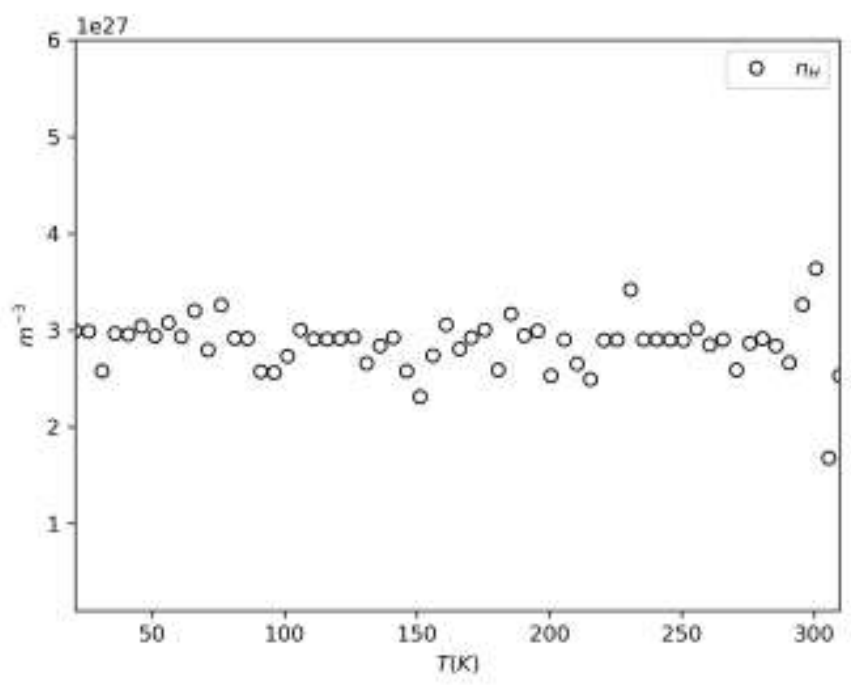

Fonte: Autores (2021).

Na Figura 9 são apresentados os resultados da mobilidade eletrônica, em $\mathrm{m}^{2} /$ Vs para a amostra de ITO. Como pode ser verificado, os dados apresentam uma pequena redução na mobilidade eletrônico a medida que a temperatura é aumentada. Esse comportamento é típico do surgimento de vibrações na rede cristalina, tais como fônon óticos e acústicos, que surgem com o 
aumento da temperatura (Oliveira, 2016). Novamente os resultados mostram que a montagem ofereceu bons resultados ao logo das medidas. Lembrando que para obtenção da concentração de portadores e da mobilidade eletrônica foi necessário realizar medidas com o campo na direção positiva, negativa e sem campo magnético, juntamente com a varação dos contatos feita pela placa apresentada na Figura 6. Todas essas operações foram controladas pela placa DAQ que era controlada por um software em Labview. O software em Labview controla todo o sistema, realiza as operações matemáticas necessárias e apresenta em tempo real os comportamentos da mobilidade, temperatura, concertação de portadores e resistividade na amostra.

Figura 9: Mobilidade eletrônica da amostra de ITO em função da temperatura. Os dados apresentam uma pequena redução na mobilidade em função do aumento da temperatura. Este comportamento é típico de espalhamento por fônons óticos e acústicos, devido ao aumento de temperatura.

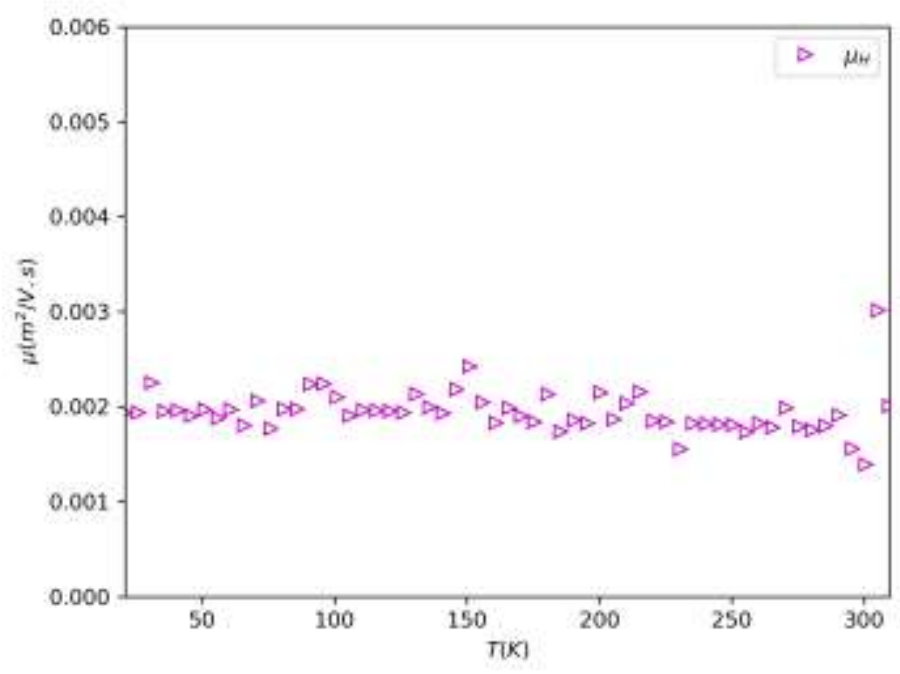

Fonte: Autores (2021).

\section{Conclusão}

Foi construída uma placa com relés para realizar as operações das medidas de mobilidade eletrônica, resistividade e concentração de portadores pela técnica de van der Pauw. Essa construção reduziu muito os custos do equipamento e serviu como objeto de aprendizagem para os alunos que participaram da construção. A automação do sistema foi realizada através de uma placa DAQ, de comunicação via GPIB, controlada por um software em Labview, que realizava o controle da temperatura, do campo magnético, das operações com os relés e da fonte e multímetro da Keithley. O software foi inteiramente construído por alunos de graduação e pós-graduação. Após essa etapa o equipamento foi testado e de acordo com os resultados as medidas foram satisfatórias. Atualmente, o equipamento está sendo utilizado para medidas de amostras de GaAs/InAs, que são pertencentes a um trabalho de doutorado e em disciplinas do programa de pós-graduação em Materiais para Engenharia da Unifei. Além disso, está disponível para a colaboração com outros pesquisadores interessados.

Diante do sucesso na construção desse experimento, nosso grupo pretende desenvolver novos equipamentos e metodologias para a caracterização elétrica e ótica de materiais. Com isso, capacitando discentes na área de instrumentação e obtendo resultados científicos, na caracterização materiais e dispositivos, relevantes para a comunidade científica.

\section{Agradecimentos}

Agradecemos o apoio financeiro da Fapemig, Cnpq e Capes. Agradecemos também a Universidade Federal de Itajubá 
(UNIFEI) e ao laboratório de Caracterização de Materiais (LCM) do Instituto de Física e Química (IFQ).

\section{Referências}

Adachi, S. (2005) Properties of Group-IV, III-V and II-VI Semiconductors. John Wiley \& Sons, Ltd

Dietrich, S., Kusnezoff, M., Petasch, U., \& Michaelis, A. (2021). Evaluation of Indium Tin Oxide for Gas Sensing Applications: Adsorption/Desorption and Electrical Conductivity Studies on Powders and Thick Films. Sensors, 21(2), 497.

Feng, Z., Qin, P., Yang, Y., Yan, H., Guo, H., Wang, X., Zhou, X., Han, Y., Yi, J., Qi, D., Yu, X., Breese, M. B. H., Zhang, X., Wu, H., Chen, H., Xiang, H., Jiang, C., \& Liu, Z. (2021). A two-dimensional electron gas based on a 5s oxide with high room-temperature mobility and strain sensitivity. Acta Materialia, 204, 116516.

Guo, H., Chu, W., Prezhdo, O. V., Zheng, Q., \& Zhao, J. (2021). Strong Modulation of Band Gap, Carrier Mobility and Lifetime in Two-Dimensional Black Phosphorene through Acoustic Phonon Excitation. The Journal of Physical Chemistry Letters, 12(16), 3960-3967.

Kraus H., Lin Q., Giustino F., Herz L. M. \& Johnston M. B. (2021) Limits to Electrical Mobility in Lead-Halide Perovskite Semiconductors. The Journal of Physical Chemistry Letters 12 (14), 3607-3617

Jiang, M. H., Wang, X. B., Xu, Q., Li, M., Niu, D. H., Sun, X. Q., Wang, F., Li, Z. Y.\& Zhang, D. M. (2018) High-speed electro-optic switch based on nonlinear polymer-clad waveguide incorporated with quasi-in-plane coplanar waveguide electrodes, Optical Materials, 75, 26-30

Liu, A. Y., Herrick, R. W., Ueda, O., Petroff, P. M., Gossard, A. C. \& Bowers, J. E., (2015) Reliability of InAs/GaAs Quantum Dot Lasers Epitaxially Grown on Silicon, in IEEE Journal of Selected Topics in Quantum Electronics, 21(6), 690-697

Look, D. C. (1992) Electrical Characterization of GaAs Materials and Devices, Wiley

Martins, G. S., Gomes, S. A. O., Louro, S. R. W., Wajnberg, E., Alves, O. C., Almeida, D. B., Cesar, C. L. \& Feder, D. (2020) Evaluation of Biological Toxicity of CdTe Quantum Dots in Trypanosoma cruzi. Research, Society and Development, 9(12), e34391211274.

Mosbah, A., Saker, A., Mekki, D. E., \& Bouzabata, B. (2021). Microstructure and Electrical Properties of the DC-sputtered Al1-xMox Alloys. Brazilian Journal of Physics, 51(3), 461-468.

Oliveira, A. F. (2015) Transporte eletrônico em amostras de InAs/GaAs e mecanismos de espalhamento. Uma nova abordagem de ajustes pelo método de otimização global. 108 f. Tese (Doutorado em Materiais para Engenharia) - Universidade Federal de Itajubá.

Oliveira, A.F., Rubinger, R.M., Monteiro, H., Rubinger C. P. L., Ribeiro G. M. \& de Oliveira A. G. (2016). Main scattering mechanisms in InAs/GaAs multiquantum-well: a new approach by the global optimization method. J Mater Sci 51, 1333-1343

Oliveira, F. S., Guimarães, L. G., dos Santos, C. A. M., de Lima, B. S., \& da Luz, M. S. (2021). Electrical and thermodynamic study of SrTiO3 reduction using the van der Pauw method. Materials Chemistry and Physics, 263, 124428.

Pereira, A. S., Shitsuka, D. M., Parreira, F. J., Shitsuka, R. (2018) Metodologia de pesquisa científica, UFSM.

Sousa, N. P., Silva, C. F., Medeiros, R. G. C., Freitas, P. G. M. \& Zenóbio, I. R. (2021) Semiconductor thin film's topographic and roughness characterization through confocal microscopy. Research, Society and Development, 10(5),e22810514833.

Sun, I. W., \& Chen, P. Y. (2014) Semiconductors Groups II-IV and III-V, Electrochemical Deposition. In: Kreysa G., Ota K., Savinell R. F. (eds) Encyclopedia of Applied Electrochemistry. Springer.

Sze, S. M., Li, Y., \& Ng, K. K. (2021) Physics of Semiconductor Devices: John Wiley \& Sons

Toledo, R. P., Huanca, D. R., Oliveira, A. F., dos Santos Filho, S. G., \& Salcedo, W. J. (2020). Electrical and optical characterizations of erbium doped MPS/PANI heterojunctions. Applied Surface Science, 529, 146994

Yong-Hang, Z., \& David, J. S. (2021) Heterovalent semiconductor structures and devices grown by molecular beam epitaxy, Journal of Vacuum Science \& Technology A 39, 030803

Wolfe, C. M., M, W. C., Wolfe, C. N., Holonyak, N., Stillman, G. E. \& Holonyak, N. J. (1989) Physical Properties of Semiconductors, Prentice Hall

Wróbel, J., Umana-Membreno, G. A., Boguski, J., Sztenkiel, D., Michałowski, P. P., Martyniuk, P., Faraone, L., Wróbel, J. \& Rogalski, A. (2020), LocallyStrain-Induced Heavy-Hole-Band Splitting Observed in Mobility Spectrum of p-Type InAs Grown on GaAs. Phys. Status Solidi RRL, 14: 1900604 